\title{
A NOTE ON STABLE HOMEOMORPHISMS OF INFINITE-DIMENSIONAL MANIFOLDS ${ }^{1}$
}

\author{
RAYMOND Y. T. WONG
}

Abstract. In papers by R. D. Anderson and R. Wong, respectively, it is shown that all homeomorphisms of the Hilbert cube onto itself, or of the infinite dimensional separable Hilbert space $l_{2}$ onto itself, are stable in the sense of Brown-Gluck. These facts can be used to show that all homeomorphisms of $X$ onto itself are isotopic to the identity mapping where $X$ is either the Hilbert cube or $l_{2}$. It follows that some versions of the infinite-dimensional annulus conjecture are true. In this note we give a simple proof of Anderson's result. It follows from Brown-Gluck's technique that for any connected manifold $X$ modeled on $Q$ or $s$, every homeomorphism of $X$ onto itself is stable.

An end-face $K$ of the Hilbert cube $Q=[0,1]^{\infty}$ is a set of the form $\pi_{i}^{-1}(0)$ or $\pi_{i}^{-1}(1)$ where $\pi_{i}$ is the projection of $Q$ onto the $i$ th-factor. We define $s=(0,1)^{\infty} \subset Q$.

Let $G(X)$ denote the set of all homeomorphisms of $X$ onto $X$ and let " $\approx$ " mean "homeomorphic to". A homeomorphism of $X$ onto itself is stable if it is the finite composition of homeomorphisms each of which is the identity on some nonempty open subset of $X$.

LEMMA. Let $h$ be any homeomorphism of $Q$ onto $Q$ and let $B$ be the union of all end-faces of $Q$, then there is an imbedding $f$ of $Q$ into s such that $f(Q) \cap h(B)=\varnothing$.

Proof. Write $B$ as $K_{1} \cup K_{2} \cup \ldots$ where each $K_{i}$ is an end-face of $Q$. Let $H$ denote the space of all imbeddings of $Q$ into $Q$ under the usual supremum metric. According to $[5$, p. 32] $H$ is completible, hence is of 2nd-category. Let $H_{i}=\left\{f^{\prime} \in H: f^{\prime}(Q) \cap\left(K_{i} \cup h\left(K_{i}\right)\right)=\varnothing\right\}$. Clearly an $f \in \bigcap_{i=1}^{\infty} H_{i}$ is the desired function if such an $f$ exists. Therefore it suffices to show that each $H_{i}$ (namely $H_{1}$ ) is both open and dense in $H . H_{1}$ is clearly open. To show $H_{1}$ is dense, suppose $\epsilon>0$ and $g \in H$. Consider $h^{-1} g(Q)$. It is clear there is a $g_{1} \in H$ such that $g_{1}\left(h^{-1} g(Q)\right) \cap K_{1}=\varnothing$. Hence $h\left[g_{1} h^{-1} g(Q)\right] \cap h\left(K_{1}\right)=\varnothing$. Again it is clear there is a $g_{2} \in H$ such that $f^{\prime}=g_{2}\left[h g_{1} h^{-1} g(Q)\right] \cap\left(K_{1} \cup h\left(K_{1}\right)\right)$ $=\varnothing$. We can now assume $g_{1}, g_{2}$ are to be chosen so small so that

Received by the editors August 12, 1969.

AMS 1969 subject classifications. Primary 5460, 5755, 5757.

Key words and phrases. Homeomorphisms, stable homeomorphisms, Hilbert cube.

1 This paper was partially written while the author held a Naval Research Grant ONR:432:LDB:lcd at the University of Washington, Seattle, and revised into its present form at the University of California, Santa Barbara. 
$\left(g_{2}\right)\left(h g_{1} h^{-1}\right) g$ is arbitrarily closed to $g$ or $d\left(f^{\prime}, g\right)<\epsilon$. This shows $H_{1}$ is dense and the Lemma is proved.

Corollary (Anderson [3]). Every $h \in G(Q)$ is stable.

Proof. It follows from the Lemma that for some sub-Hilbert cube $K \subset s, h(K) \subset s$. By [1] there are $f, g \in G(Q)$ with $f, g$ stable, $f$ equal to $h^{-1}$ on $h(K)$ and with $g(K)=K_{1}$, where $K_{1}=\pi_{1}^{-1}(0)$. Then $g f h g^{-1}$ is the identity on $K_{1}$. It follows that $g f h g^{-1}$ is stable (Wong proves it for $s$ [6], similar result for $Q$, Anderson [3, Lemma 3.1]). Hence $h$ is stable.

The author thanks the referee for pointing out an alternative (but simpler) proof of the following theorem, whose version is adopted here.

TheOREM. Let $X$ be a connected manifold modeled on $Q$ or $s$, then every $h \in G(X)$ is stable.

Proof. Let $p \in X$. Following the standard device of forming simple chains from $p$ to $h(p)$, there is a stable homeomorphism $g$ of $X$ onto itself such that $g(h(p))=p$. Thus for simplicity we may assume $h(p)$ $=p$. Let $U, V$ be basic open sets in $X$ with $p \in V \subset \mathrm{Cl}(V) \subset U$, with $\operatorname{Bd}(U) \approx \operatorname{Bd} V \approx Q$ or $s$, and with $h(V) \subset U$. Then by Corollary 10.6 of [3] (for $Q$ ) and a similar argument for $s$ (using [6]), $\mathrm{Cl}(U) \backslash V$ $\approx \mathrm{Cl}(U) \backslash h(V) \approx Q$ (or $s)$. But since $\mathrm{Bd} U \cup \mathrm{Bd} V, \mathrm{Bd} U \cup \mathrm{Bd} h(V)$ are $Z$-sets of $\mathrm{Cl}(U) \backslash V$ and $\mathrm{Cl}(U) \backslash h(V)$ respectively, by Theorem 10.1 of [3] there is a homeomorphism $f$ of $\mathrm{Cl}(U) \backslash h(V)$ onto $\mathrm{Cl}(U) \backslash V$ which is the identity on $\mathrm{Bd} U$ and is $h^{-1}$ on $h(\mathrm{Bd} V)$. Let $F$ be the extension of $f$ to $X$ onto itself which is the identity on $X \backslash U$ and is $h^{-1}$ on $h(V)$. Then $F h$ is the identity on $V$. Since $h=F^{-1}(F h), h$ is stable.

\section{REFERENCES}

1. R. D. Anderson, Topological properties of the Hilbert cube and the infinite product of open intervals, Trans. Amer. Math. Soc. 126 (1967), 200-216. MR 34 \#5045.

2. - Hilbert space is homeomorphic to the countable infinite product of lines, Bull. Amer. Math. Soc. 72 (1966), 515-519. MR 32 \#8298.

3. —_ On topological infinite deficiency, Michigan J. Math. 14 (1967), 365383. MR 35 \#4893.

4. M. Brown and H. Gluck, Stable structures on manifolds. I. Homeomorphisms of $S^{n}$, Ann. of Math. (2) 79 (1964), 1-17. MR 28 \#1608a.

5. C. Kuratowski, Topologie. Vol. 2, 3rd ed., Monografie Mat., Tom 21, PWN, Warsaw, 1961, p. 32 (7); English transl., Academic Press, New York (to appear). MR 24 \#A2958.

6. R. Y. T. Wong, On homeomorphisms of certain infinite dimensional spaces, Trans. Amer. Math. Soc. 128 (1967), 148-154. MR 35 \#4892.

University of Washington, Seattle, Washington 98105

University of California, Santa Barbara, California 93106 